\title{
Density Distribution for a Polymer Adsorbed at an Oil-Water Interface
}

\author{
J. Cai and J.M. Prausnitz* \\ Department of Chemical Engineering, University of California, Berkeley and \\ Chemical Sciences Division, Lawrence Berkeley National Laboratory, Berkeley, CA94720
}

\begin{abstract}
The interaction between a polymer segment and an oil-water interface is represented by an asymmetric square-well potential where the well-depth on one side reflects waterpolymer and the well depth on the other side reflects oil-polymer interactions. The polymer is represented by a Gaussian chain. The polymer's density distribution is calculated along a coordinate perpendicular to the interface. Results are obtained as a function of the well width, the well depth and its asymmetry and, most important, the polymer's length. For a symmetric well, the distribution shows a strong maximum at the interface provided that the polymer is sufficiently long. For an asymmetric well, the polymer is also strongly adsorbed at the interface provided that the polymer is sufficiently long and provided that the larger well-depth does not exceed a critical value that depends on the smaller well-depth. The calculations are in substantial agreement with experimental results that indicate nearly irreversible adsorption of long-chain molecules at an oil-water interface.
\end{abstract}

Experimental results $[1,2]$ show that when a long flexible polymer chain adsorbs on the oil-water interface, it is adsorbed so strongly that, essentially, it remains at the interface indefinitely; this phenomenon, discussed by De Gennes over 20 years ago [3], has received wide attention [4]. One theory to explain this phenomenon is Gaussian-chain theory that leads to a diffusion or Schroedinger-like equation [5]. For the simplest case (no interaction between segments other than bond energy), we obtain the general solution of the diffusion equation[6] that is a function of eigenvalues. For the more general case, perturbation methods or numerical methods must be used to obtain an asymptotic solution [4,7] or numerical results [8]. Most previous work has been confined to the longchain systems where the ground state is dominant. In this work, we use the Gaussian-

\footnotetext{
* To whom correspondence should be addressed
} 
chain model to show how the extent of adsorption is quantitatively related to the length of the polymer chain and to the strength of interaction between the polymer and the two sides of a liquid-liquid interface.

Consider a linear polymer chain with $N$ segments dissolved in a continuous medium. The configurational partition function of the polymer [5] is given by

$$
Z=\int \exp \left[-\beta V_{\text {ext }}\left(\left\{\mathbf{r}_{j}\right\}\right)-\beta U_{\text {intra }}\left(\left\{\mathbf{r}_{j}\right\}\right)\right] \mathrm{d}\left\{\mathbf{r}_{j}\right\}
$$

where $\beta=1 / k_{\mathrm{B}} T ; T$ is temperature; $k_{\mathrm{B}}$ is Boltzmann constant; $\mathbf{r}_{j}$ is the coordinate of the $j$-th segment of the chain and $\left\{\mathbf{r}_{j}\right\}$ represents the set of all position coordinates of the segments. $V_{\text {ext }}$ is the external potential and $U_{\text {intra }}$ is the segment-segment potential including the segment-segment bonding energy. Because we want to examine adsorption of the polymer at the liquid-liquid interface, we do not consider details of the potential between the polymer segments. The polymer is represented by an ideal Gaussian chain without any interaction between the non-bonded segments; only the segment-segment bonding energy is considered. As shown elsewhere [5], it is given by

$$
\exp \left[-\beta U_{\text {intra }}\left(\left\{\mathbf{r}_{j}\right\}\right)\right]=\prod_{j=1}^{N-1}\left(\frac{3}{2 \pi l \Delta s}\right)^{3 / 2} \exp \left[-\frac{3\left(\mathbf{r}_{j+1}-\mathbf{r}_{j}\right)^{2}}{2 l \Delta s}\right]
$$

where $l$ is the statistical segment length; polymer length $L$ equals $N \cdot \Delta s$. Because temperature does not appear in the right side of Eq. (2), the configuration of the polymer in the bulk is independent of temperature. The polymer is placed in a liquid system containing an oil-water interface. We calculate how the polymer is distributed in the liquid in the direction perpendicular to the surface. Since the oil is hydrophobic, molecules of oil and water are strongly repulsive. When the polymer goes into the oilwater interface, some of the oil and water molecules at the interface do not contact directly, decreasing the surface energy. Thus, the oil-water surface is like a potential well for the polymer. Because only adsorption behavior is of interest here, we do not explicitly consider interactions among oil, water and polymer but consider only interactions of the interface with the polymer. We model this surface energy as an asymmetric square-well and the bulk phases oil and water as two continuous media. The oil-water interface is in the $x-y$ plane; coordinate $\mathrm{z}$ is perpendicular to that plane. The external potential of a chain segment of unit length is given by $v(z)$ : 


$$
\beta v(z)=\left\{\begin{array}{c}
+\infty ; \quad z<-H_{1} \text { or } z>H_{2} \\
\beta\left(\varepsilon_{1}-\varepsilon_{0}\right) ; \quad-H_{1} \leq z \leq-a / 2 \\
-\beta \varepsilon_{0} ; \quad-a / 2<z<a / 2 \\
0 ; \quad a / 2 \leq z \leq H_{2}
\end{array}\right.
$$

As shown in Fig. $1, \varepsilon_{0}, \varepsilon_{1} \geq 0$ are the well depths, one on the water side and the other on the oil side; $a$ is the well width and $H_{1}+H_{2}$ is the width of the system in the $z$ direction.

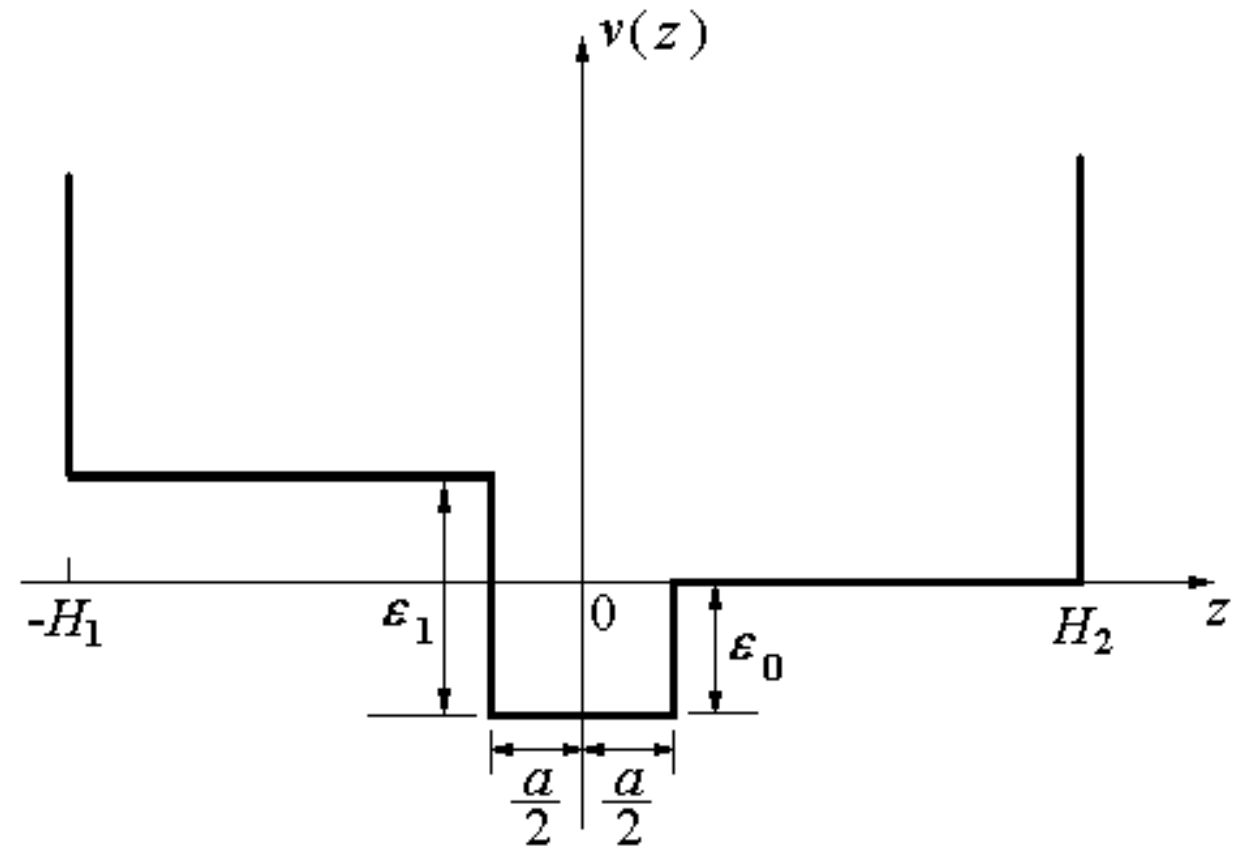

Fig. 1 The potential of the system

From Eqs. (1) and (2), the configurational partition function of the system becomes

$$
Z=\left(\frac{3}{2 \pi l \Delta s}\right)^{3 N / 2} \int \exp \left[-\frac{3}{2 l \Delta s} \sum_{j=1}^{N-1}\left(\mathbf{r}_{j+1}-\mathbf{r}_{j}\right)^{2}-\Delta s \sum_{j=1}^{N} \beta v\left(z_{j}\right)\right] \mathrm{d}\left\{\mathbf{r}_{j}\right\}
$$

where $\mathbf{r}_{j}$ is the position vector of the $j$-th segment of the polymer. To obtain the partition function $Z$ in Eq. (4), we follow the method of Freed [5]. First we consider the case where the coordinates of both ends of the polymer are fixed. The partition function with two fixed ends $G\left(\mathbf{r}, \mathbf{r}^{\prime} ; L\right)$ is given by: 


$$
\begin{aligned}
G\left(\mathbf{r}, \mathbf{r}^{\prime} ; L\right) & =\left(\frac{3}{2 \pi l \Delta s}\right)^{3 N / 2} \int \delta\left(\mathbf{r}_{1}-\mathbf{r}\right) \delta\left(\mathbf{r}_{N}-\mathbf{r}^{\prime}\right) \times \\
& \times \exp \left[-\frac{3}{2 l \Delta s} \sum_{j=1}^{N-1}\left(\mathbf{r}_{j+1}-\mathbf{r}_{j}\right)^{2}-\Delta s \sum_{j=1}^{N} \beta v\left(z_{j}\right)\right] \mathrm{d}\left\{\mathbf{r}_{j}\right\}
\end{aligned}
$$

where $\delta(\mathbf{r})$ is the Dirac function. Since the $x$ and $y$ coordinates are of no concern, we rewrite Eq. (5) in the form

$$
\begin{aligned}
G\left(z, z^{\prime} ; L\right)=\left(\frac{3}{2 \pi l \Delta s}\right)^{N / 2} & \int \mathrm{d} z^{N} \delta\left(z_{1}-z\right) \delta\left(z_{N}-z^{\prime}\right) \times \\
& \times \exp \left[-\frac{3}{2 l \Delta s} \sum_{j=1}^{N-1}\left(z_{j+1}-z_{j}\right)^{2}-\Delta s \sum_{j=1}^{N} \beta v\left(z_{j}\right)\right] .
\end{aligned}
$$

$G\left(z, z^{\prime} ; L\right)$ represents the correlation between two end points of the polymer. In the flexible-chain limit, i.e. $\Delta s \rightarrow 0$, it can be shown [3-5] that the function $G\left(z, z^{\prime} ; L\right)$ satisfies the differential equation:

$$
\frac{\partial G}{\partial L}-\frac{l}{6} \frac{\mathrm{d}^{2} G}{\mathrm{~d} z^{2}}+\beta v(z) G=0 .
$$

The boundary condition is

$$
G\left(z, z^{\prime} ; L\right)=0 ; \quad z, z^{\prime} \leq 0 \text { or } \geq H .
$$

The initial condition when $L$ tends to zero (similar to an ideal-gas particle) is given by

$$
\lim _{L \rightarrow 0} G\left(z, z^{\prime} ; L\right)=\delta\left(z-z^{\prime}\right) .
$$

The general solution of Eq. (7) is well known [3]. Eq. (7) is a diffusion equation equivalent to the Schrödinger equation. Details of the solution are given in the Appendix.

The final solution of differential equation Eq. (7) is

$$
G\left(z, z^{\prime} ; L\right)=\sum_{k=0}^{+\infty} u_{k}(z) u_{k}\left(z^{\prime}\right) \exp \left(-\beta L E_{k}\right)
$$

where $u_{k}(z)$ is the normalized eigenfunction and $E_{k}$ stands for the eigenvalues of energy satisfying $E_{0}<E_{1}<\cdots$. To obtain the density distribution of the segments in the $z$ direction, it is convenient to separate the polymer chain into two parts: $L_{1}$ and $L-L_{1}$. The density distribution is given by

$$
\rho(z)=Z^{-1} L^{-1} \int_{-H_{1}}^{H_{2}} \mathrm{~d} z_{1} \int_{-H_{1}}^{H_{2}} \mathrm{~d} z_{2} \int_{0}^{N} G\left(z_{1}, z ; L_{1}\right) G\left(z, z_{2} ; L-L_{1}\right) \mathrm{d} L_{1} .
$$


According to Eq. (6), the partition function $Z$ is given by

$$
Z=\int_{-H_{1}}^{H_{2}} \mathrm{~d} z_{1} \int_{-H_{1}}^{H_{2}} \mathrm{~d} z_{2} G\left(z_{1}, z_{2} ; L\right) .
$$

Since the multiplier $L^{-1}$ in Eq. (11), $\rho(z)$ is defined as the density distribution of the unit polymer length. The integral of $\rho(z)$ over the entire system is unity, i.e.

$$
\int_{-H_{1}}^{H_{2}} \rho(z) \mathrm{d} z=1 .
$$

If the chain length is very large, the only important term is the first term of Eq. (10), i.e. the lowest-energy state or the ground state. In the limit of an infinitely long chain, the density distribution $\rho_{\infty}$ is given by

$$
\rho_{\infty}(z)=u_{0}^{2}(z)
$$

where subscript $\infty$ represents the infinitely long chain. Well depths $\varepsilon_{0}$ and $\varepsilon_{1}$ determine the choice of Eq. (A8) or Eq. (A13) or Eq. (A16) for the ground state. If the ground state is expressed by Eq. (A8), the sufficiently long polymer adsorbs strongly at or near the interface. If the ground state is expressed by Eq. (A13), the sufficiently long polymer prefers to stay in one phase, partly adsorbs at the surface and distributes uniformly in the liquid phase of lower potential energy. Eq. (A16) indicates that the polymer distribution is more uniform than that given by Eq. (A8) or (A13) throughout the entire two-phase system.

We use Eq. (11) to investigate how the potential well affects adsorption of the polymer at the interface. (Equations for the configurational entropy and energy are given in the Appendix.) The statistical segment length $l$ is chosen to be a length unit, i.e. $l=1$. The well is located at the center of the box in the $z$-axis direction such that the center of the well is at $z=0$. We use a numerical method for finding the eigenvalues of Eq. (7). We use the first 1000 terms to approximate the infinite series in Eq. (10). Since the exponential term in Eq. (10), 1000 terms are enough to ensure the accuracy. To ensure that the walls of the container do not influence the density distribution near the interface, the width of the system is set to a large number $200 l$ where $H_{1}=H_{2}=100 l$. Our results indicate that this value of width is sufficiently large; larger values of $H_{1}$ and $H_{2}$ do not affect the density distribution close to limiting distribution but make the value of density distribution of short chain smaller. Fig. 2 shows how the width of the container influences 
the density distribution. The potential well is symmetric, $\beta \varepsilon_{1}=\beta \varepsilon_{0}=1$. The width of the well $a=2 l$. The width of the system in the $z$ direction varies from $100 l$ to $400 l$. When the walls of system is far from the interface, the system is large, a short chain distributes uniformly in the system. But for a sufficiently long chain, it adsorbed strongly at the surface, the distribution is not affected by the distance between walls and interface.

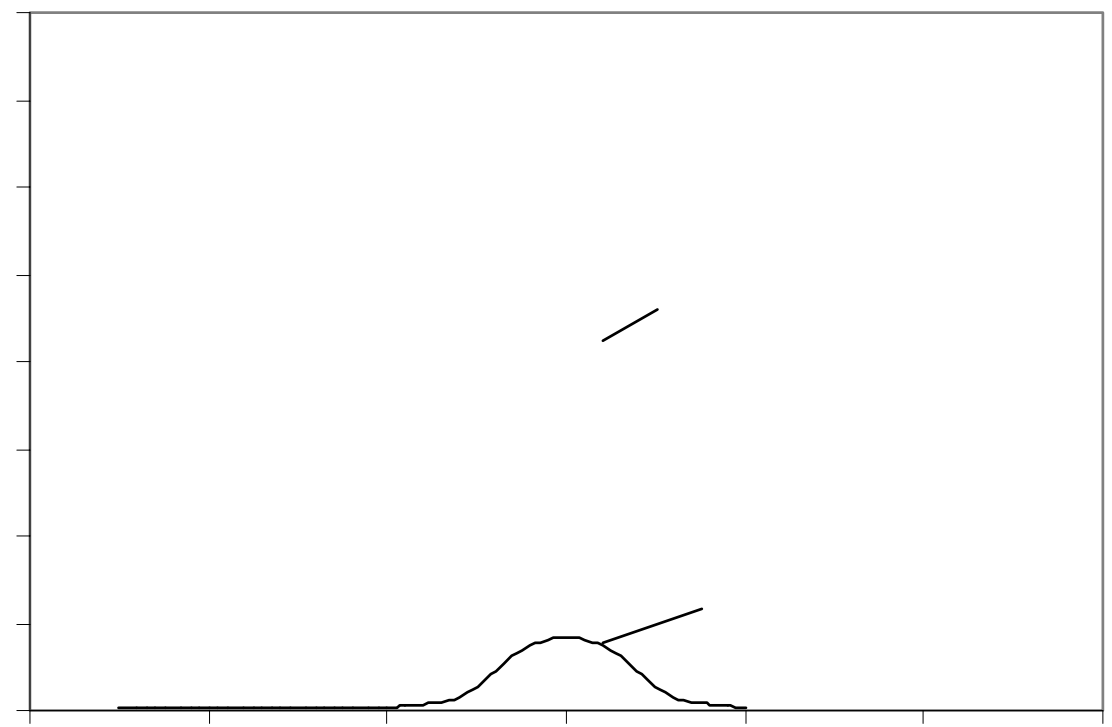

Fig. 2 Effect of the width of system on polymer-density distribution in the symmetric well with $a / l=2, \beta \varepsilon_{0}=\beta \varepsilon_{1}=1$. For a short chain $(L=4 l)$, the density distribution is affected by the distance between the wall and the interface, but for a long chain $(L=64 l)$, the effect is negligible.

If the potential well is symmetric, i.e. $\varepsilon_{1}=\varepsilon_{0}$, there always exists at least one solution of Eq. (A8) when the energy is less than zero, no matter how shallow the well, because in this case there is at least one root of Eq. (A7). For a sufficiently long polymer, the density distribution function is like an exponential function [see Eq. (A8)] that decreases quickly when far from the well. The polymer adsorbs so strongly that it tends to remain at the interface. If the polymer is short, the effects of the second and the following terms in the infinite series of Eq. (10) cannot be omitted. According to Eq. 
(A16), the eigenfunctions are periodic, indicating that the finite-length polymer tends to distribute more uniformly in the entire system than the infinitely long polymer. Fig. 3 shows the dependence of the distribution on polymer length. In Fig. 3a, for a symmetric well, the width and depth of the well are $a / l=2$ and $\beta \varepsilon_{0}=1$. Fig. $3 \mathrm{~b}$ shows a local enlargement of Fig. 3a. When the chain length is very short, the distribution tends to be nearly flat. But for intermediate and long chains, Figs. 2 and 3 show that the distribution function is nearly zero outside the well; in other words, for such chains, adsorption at the interface is very strong.

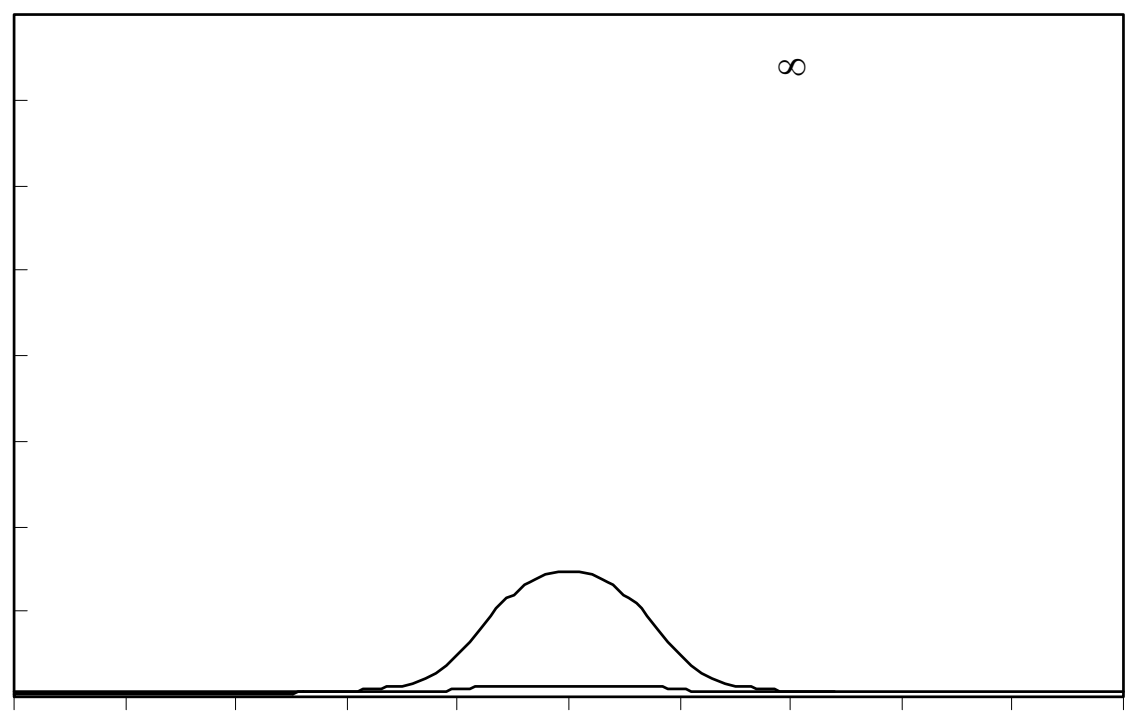

Fig. 3a Effect of polymer length on polymer-density distribution in the symmetric well with $a / l=2, \beta \varepsilon_{0}=\beta \varepsilon_{1}=1$. 


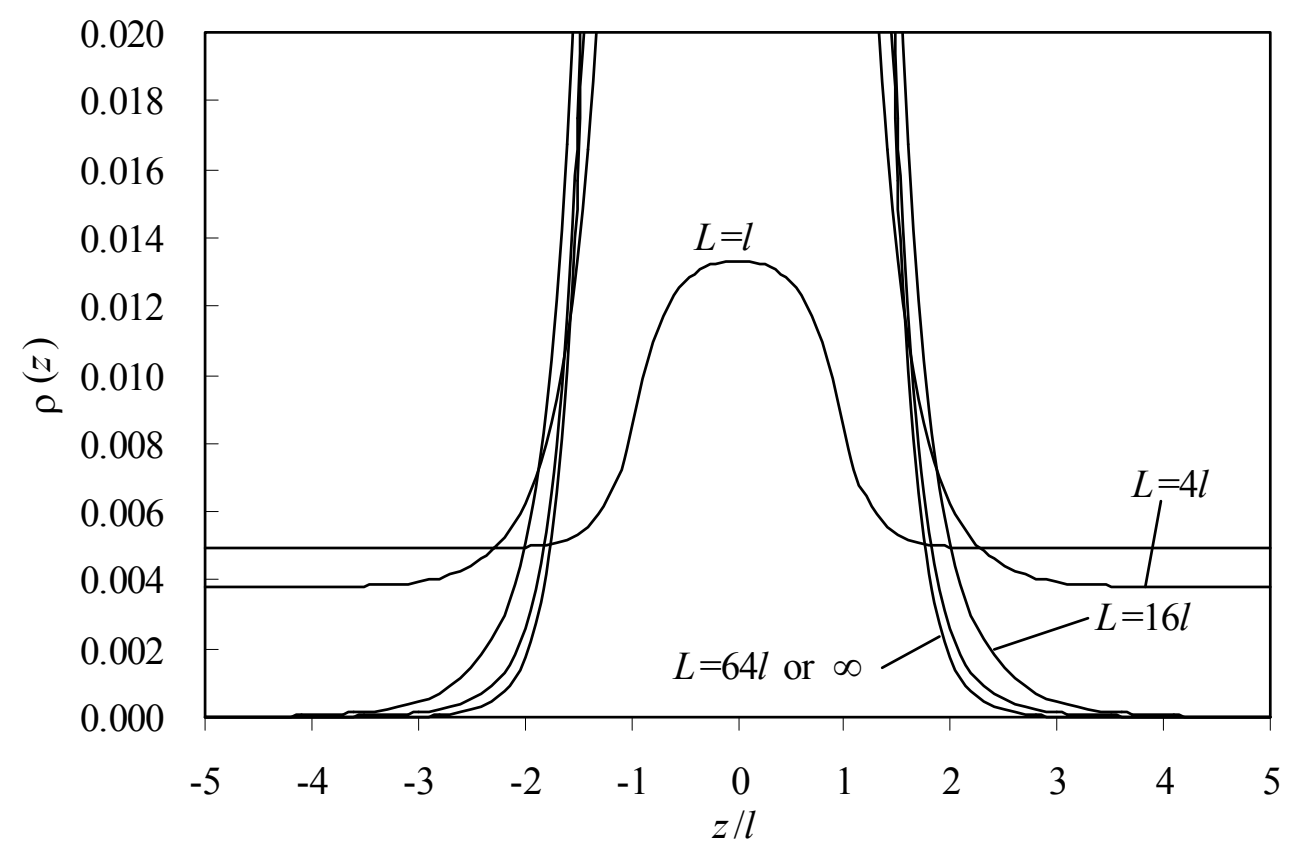

Fig. 3b Local enlarged view of Fig. 2.

If the potential well is asymmetric i.e. $\varepsilon_{1}>\varepsilon_{0}$, we first consider the limit $\varepsilon_{1}=\infty$, that is, a hard wall at $z=-a / 2$. This case corresponds to the condition that the polymer is insoluble in one of the two liquid phases; in contrast to the symmetric case where $\varepsilon_{1}=\varepsilon_{0}$ described in Figs. 2 and 3, the existence of a solution of Eq. (A7) depends on the depth of the square well $\varepsilon_{0}$. This is because of the strongly repulsive interaction due to the hard wall. The transition from the case where a solution to Eq. (A7) exists to one where it does not, occurs where the ground state energy $E_{0}=0$. The value of $\varepsilon_{0}$ corresponding to $E_{0}=0$, denoted by $\varepsilon_{0, \text { tran }}$, is the zero point of the equation:

$$
\sqrt{6 \beta \varepsilon_{0, \text { tran }} / l} \cos \left(a \sqrt{6 \beta \varepsilon_{0, \text { tran }} / l}\right)+\sin \left(a \sqrt{6 \beta \varepsilon_{0, \text { tran }} / l}\right) /\left(H_{2}-a / 2\right)=0 .
$$

When $\varepsilon_{0}$ is smaller than $\varepsilon_{0 \text {,tran }}$, there is no solution of Eq. (A7) indicating that the polymer distributes uniformly in the system. Fig. 4 shows the dependence of the density distribution for a very long chain on the depth of the square well closed to a hard wall. The value of $\varepsilon_{0 \text {,tran }}$ is $\beta \varepsilon_{0, \text { tran }}=0.104485$ for $a / l=2$. When $\varepsilon_{0}$ is larger than $\varepsilon_{0 \text {,tran }}$, the chain tends to stay in the well. Figure 5 shows how the distribution function is related to 
the well width. For clarity, the results of Fig. 5 are translated by $a / 2$ along the $z$-axis such that the hard wall is at $z=0$. When the width increases, the polymer tends to stay in the well.

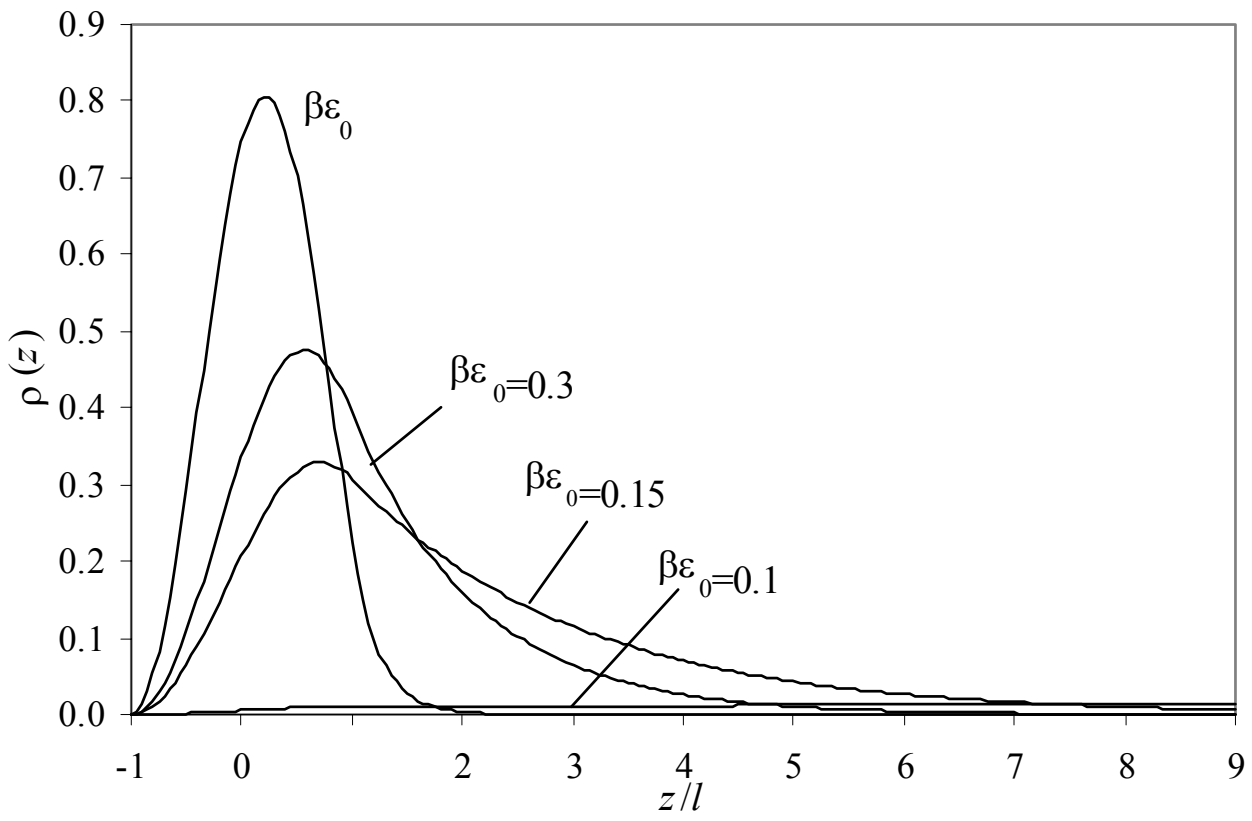

Fig. 4 Effect of energy $\varepsilon_{0}$ on the density distribution of a polymer in the square well.

Chain length $L=\infty$; width of the well $a / l=2 ; \varepsilon_{1}=+\infty$ (hard wall) 


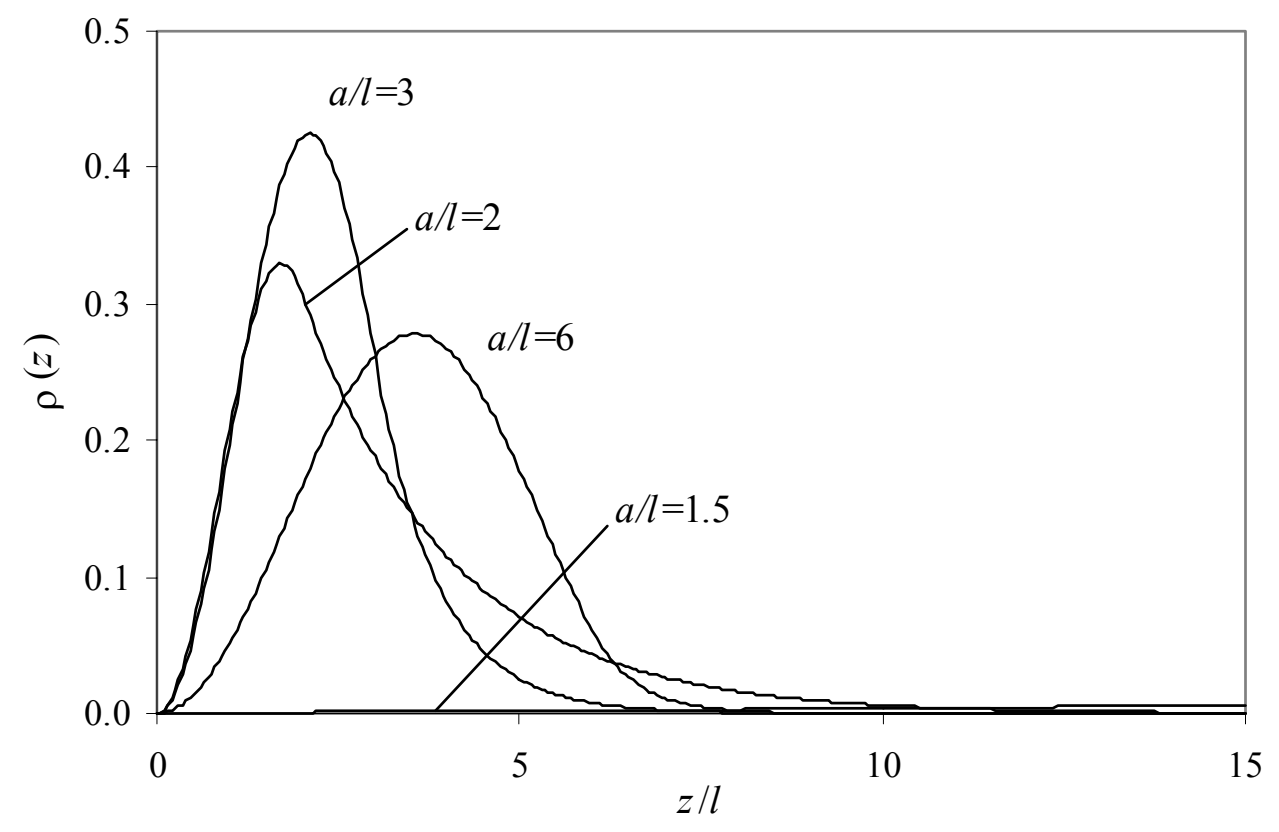

Fig. 5 Effect of well width on the density distribution of a polymer in the square well.

Chain length: $L=\infty$; potential of the well $\beta \varepsilon_{0}=0.15 ; \varepsilon_{1}=+\infty$ (hard wall).

Because the horizontal axis has been translated by $a / 2$, the hard wall is at $z=0$

For the intermediate condition $\varepsilon_{0}<\varepsilon_{1}<+\infty$, the existence of a solution of Eq. (A7) depends on $\varepsilon_{1}$ when $\varepsilon_{0}$ is given. The transition value of $\varepsilon_{1}$ denoted by $\varepsilon_{1, \text { tran }}$, similar to Eq. (15), is the solution of the equation

$$
\begin{aligned}
& \frac{\sqrt{6 \beta \varepsilon_{0} / l} \tanh \left[\left(H_{1}-a / 2\right) \sqrt{6 \beta\left(\varepsilon_{1, \text { tran }}-\varepsilon_{0}\right) / l}\right]+\sqrt{6 \beta\left(\varepsilon_{1, \text { tran }}-\varepsilon_{0}\right) / l} \tan \left(a \sqrt{6 \beta \varepsilon_{0} / l}\right)}{-\sqrt{6 \beta \varepsilon_{0} / l} \tanh \left[\left(H_{1}-a / 2\right) \sqrt{6 \beta\left(\varepsilon_{1, \text { tran }}-\varepsilon_{0}\right) / l}\right] \tan \left(a \sqrt{6 \beta \varepsilon_{0} / l}\right)+\sqrt{6 \beta\left(\varepsilon_{1, \text { tran }}-\varepsilon_{0}\right) / l}} \\
& =-\sqrt{6 \beta \varepsilon_{0} / l}\left(H_{2}-a / 2\right) .
\end{aligned}
$$

When $\varepsilon_{1}$ is larger than $\varepsilon_{1, \text { tran }}$, there is no solution of Eq. (A7) indicating that the polymer adsorbs partly at the interface. Fig. 6 shows how the well depth of left-hand side $\varepsilon_{1}$ affects the density distribution of a very long polymer. The well width is $a / l=2$; the well depth of the right-hand side $\beta \varepsilon_{0}=0.1$; and chain length $L=+\infty$. When $\varepsilon_{1}$ is larger than $\varepsilon_{1, \text { tran }}$, the density distribution is nearly flat indicating that the polymer distributes uniformly in the system. 


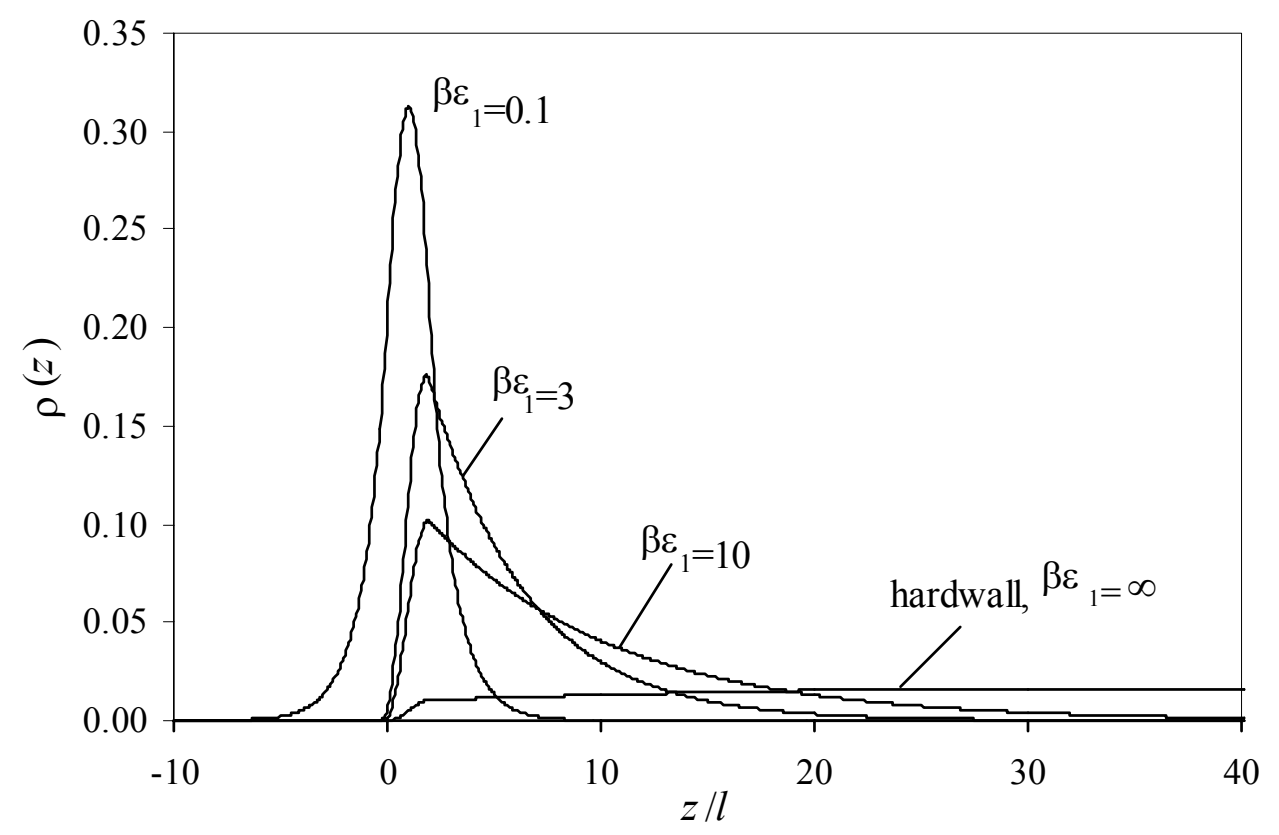

Fig. 6 Effect of $\varepsilon_{1}$ on the density distribution of a polymer in an asymmetric square well.

Well width $a / l=2$, potential of the well $\beta \varepsilon_{0}=0.1$, chain length $L=+\infty$.

Fig. 7 shows the effect of polymer length on density distribution. The width of the well is $a / l=2$ and the depth of the well is $\beta \varepsilon_{0}=0.1$ and $\beta \varepsilon_{1}=0.6$. Figs. $8 \mathrm{a}$ and $8 \mathrm{~b}$ show a local enlargement of Fig. 7. A short polymer prefers to stay in the liquid phase of lower energy but a sufficiently long polymer prefers to stay in the well provided that the ground state can be expressed by Eq. (A8). In this case, when the chain length is larger than 4096, the density distribution is essentially the same as that of the infinitely long chain. 


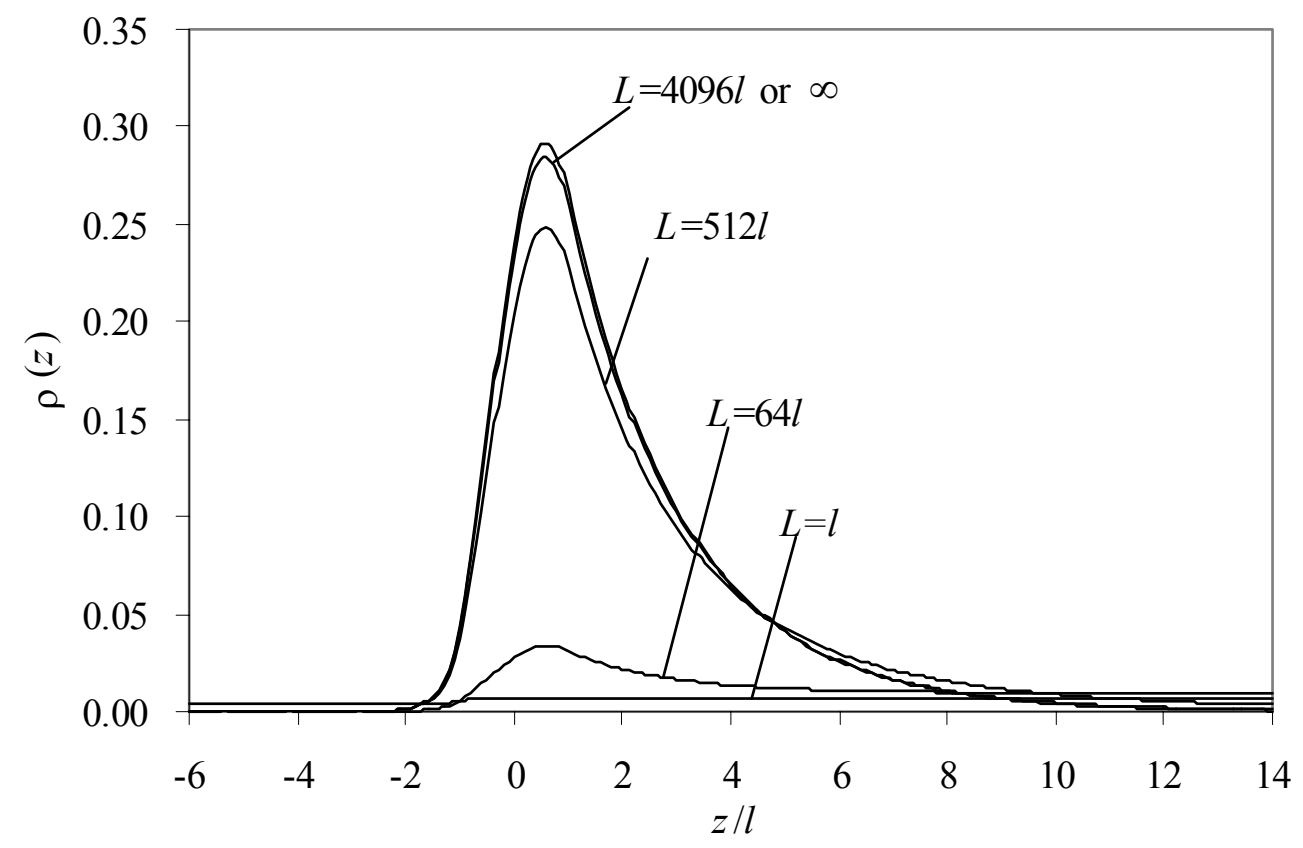

Fig. 7 Effect of polymer length on its density distribution in an asymmetric square well.

Width of well: $a / l=2$, potential of well: $\beta \varepsilon_{0}=0.1, \beta \varepsilon_{1}=0.6$.

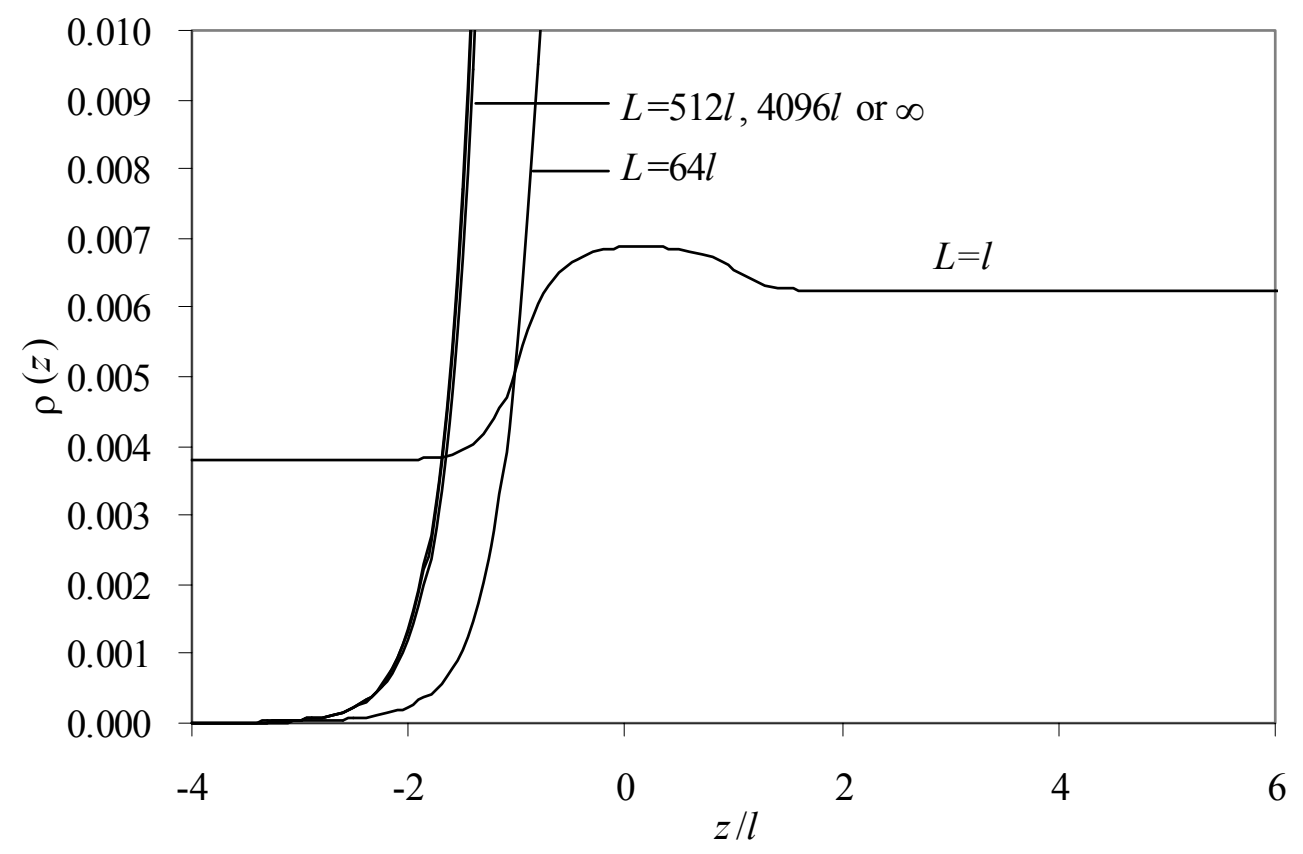

Fig. 8a Local enlarged view of Fig. 7 for small $z / l$. 


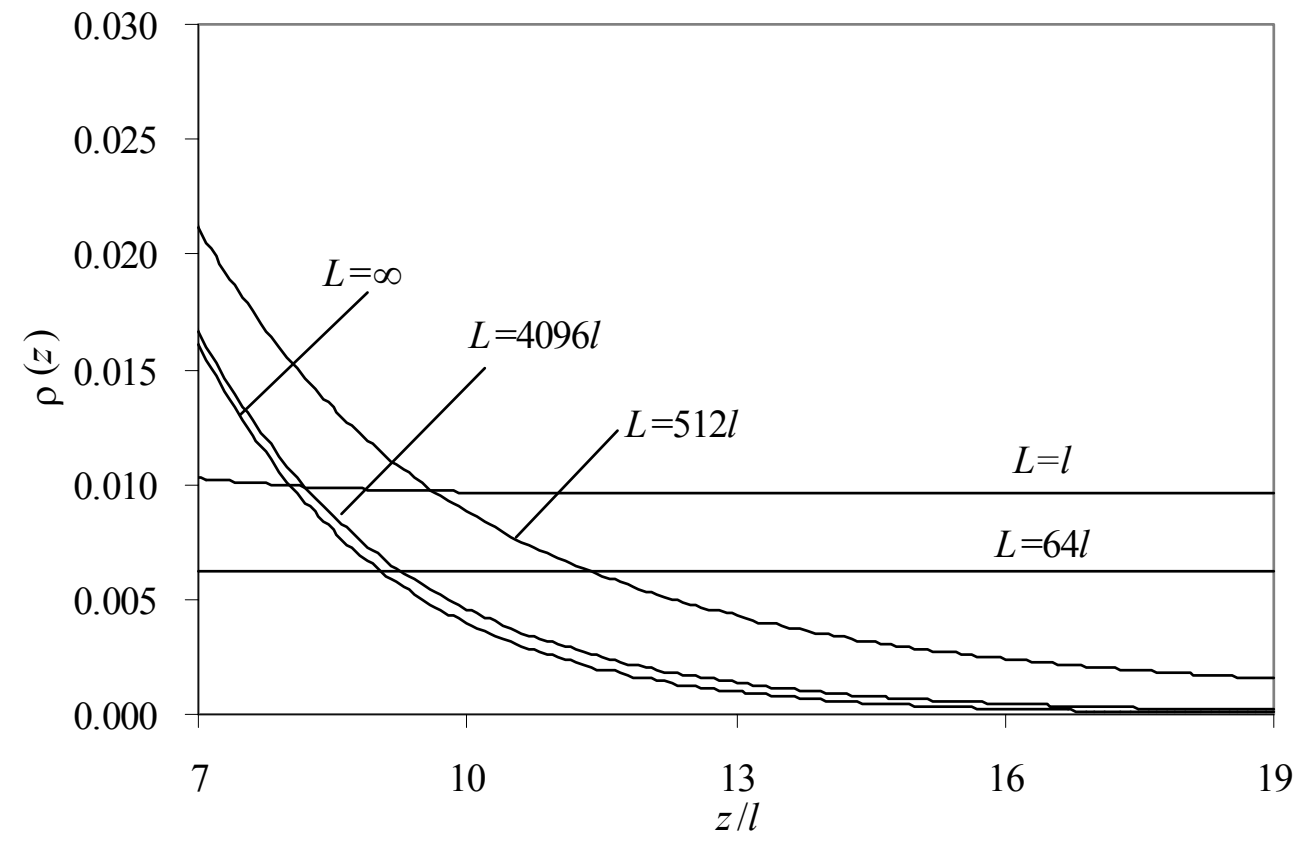

Fig. 8b Local enlarged view of Fig. 7 for large $z / l$.

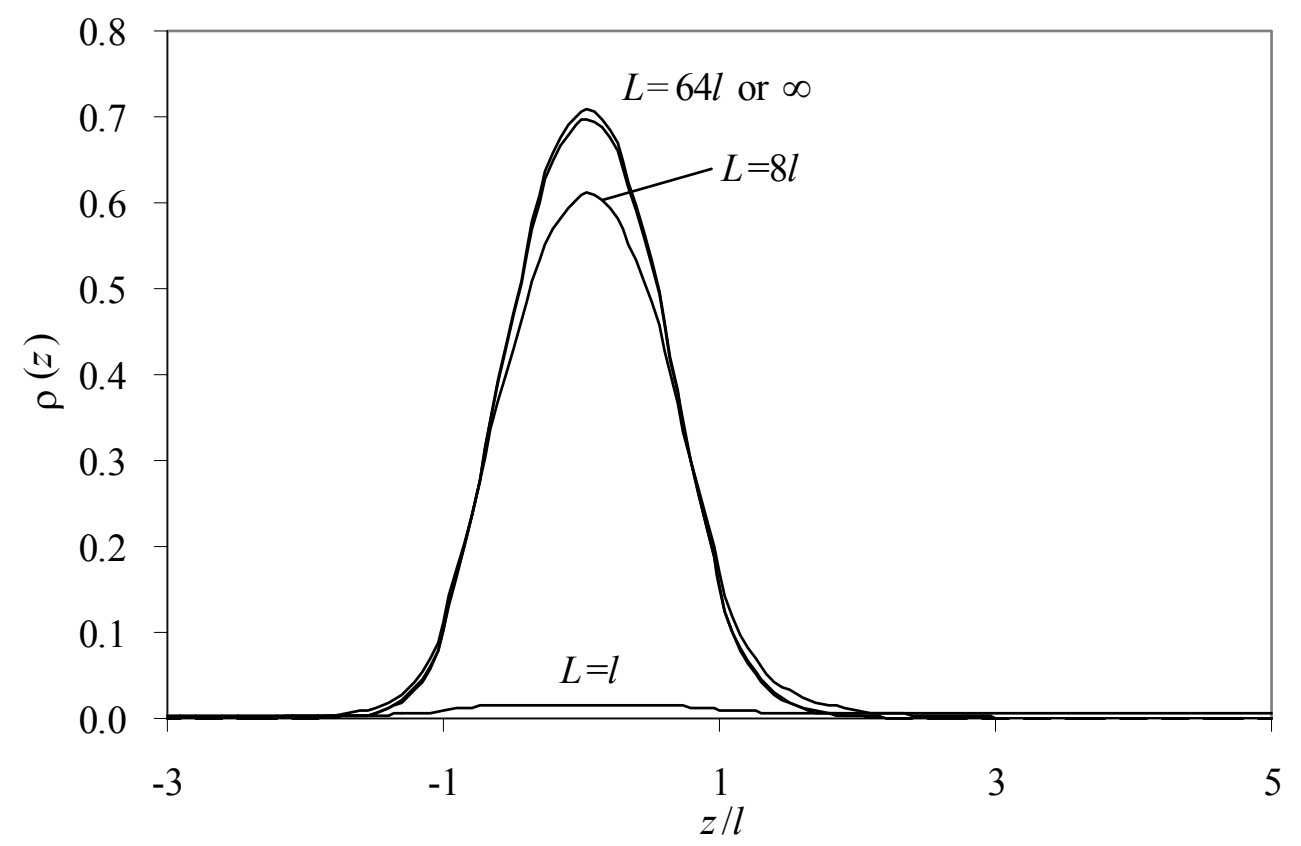

Fig. 9 Density distribution in an asymmetric well.

Width of well: $a / l=2$, potential of well: $\beta \varepsilon_{0}=1, \beta \varepsilon_{1}=1.5$. 


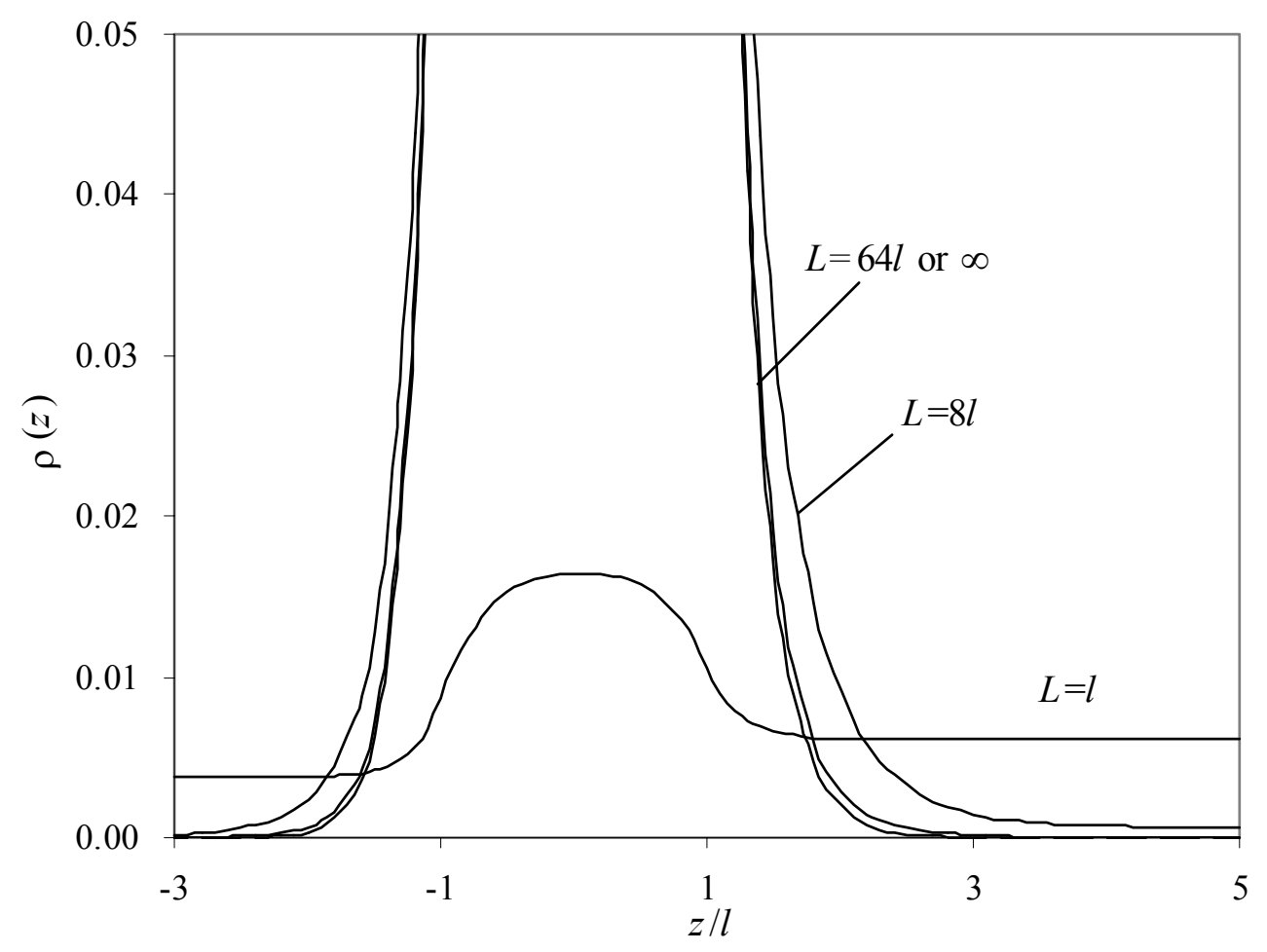

Fig. 10 Local enlarged view of Fig. 9.

Fig. 9 is similar to Fig. 7 but with a deeper well, $\beta \varepsilon_{0}=1$. Fig. 10 is a local enlarged view of Fig. 9. In this case, when the chain length is larger than 64, the density distribution is almost the same as that for a chain of infinite length. But in Fig. 7, this case occurs when the chain length is larger than 4096. The difference between Fig. 7 and Fig. 9 shows that in a deeper well, the limiting $(L=\infty)$ distribution is realized for shorter chains.

According to the discussion above, we obtain insight into the adsorption behavior at an oil-water interface. When the liquid-liquid interface is modeled as a square well, calculated results are in good agreement with experiment [1,2]. The adsorption of a polymer at the interface depends on the chain length and on the depth and width of the potential well. A short chain distributes more uniformly than a long chain. When the well depth is larger than a certain value, a sufficiently long chain prefers to stay in the well indicating that a sufficiently long polymer adsorbed at the oil-water interface is essentially adsorbed irreversibly. 


\section{Literature Cited:}

1. M. Tupy, H. Blanch, C. Radke, Ind. Eng. Chem. Res., 37 (2001) 3159-3168

2. C. Beverung, C. Radke, H. Blanch, Biophys. Chem., 81(1999)59-80

3. See P. G. de Gennes, Macromolecules, 14(1981)1637-1644 and references cited there

4. See e.g. E. Eisenriegler, Polymers Near Surfaces, World Scientific, Singapore, 1993

5. K. Freed, Renormalization Group Theory of Macromolecules, John Wiley and Sons, New York, 1987

6. P. de Gennes, Scaling Concepts in Polymer Physics, Cornell University Press, Ithaca and London, 1979

7. S. Stepanow, J. Chem. Phys., 115 (2001) 1565-1568

8. I. Teraoka, P. Cifra, J. Chem. Phys., 115 (2001) 11362-11370

\section{Acknowledgement}

For financial support, the authors are grateful to the Office for Basic Sciences, US Department of Energy and to the Chinese National Science Foundation.

The following Appendix need not be published. It can be placed in an document depository or on the internet. 


\section{Appendix:}

Derivation of Eq. (10)

Eq. (7) can be variable-separated. First let

$$
G\left(z, z^{\prime} ; L\right)=g\left(z, z^{\prime}\right) f(L)
$$

where $g\left(z, z^{\prime}\right)$ is a function of $z$ and $z^{\prime}$ only, while $f(L)$ is a function of $L$ only. We then obtain

$$
\frac{1}{f(L)} \frac{\partial f(L)}{\partial L}=-\beta E=\frac{l}{6 g\left(z, z^{\prime}\right)} \frac{\mathrm{d}^{2} g\left(z, z^{\prime}\right)}{\mathrm{d} z^{2}}-\beta v(z)
$$

where $E$ is a constant with dimensions of energy per unit polymer length. An integration of the first equality of Eq. (A2) shows that the function $f(L)$ is given by

$$
f(L)=\exp (-\beta L E) .
$$

The second equality of Eq. (A2) is a $2^{\text {nd }}$-order linear differential equation. First we must find its eigenvalues of energy $E$ and eigenfunctions. For $E$ less than zero, for the sake of convenience, let

$$
k_{0}^{2}=6 \beta\left(E+\varepsilon_{0}\right) / l, \quad k_{1}^{2}=6 \beta\left(-E+\varepsilon_{1}-\varepsilon_{0}\right) / l, \quad k_{2}^{2}=-6 \beta E / l .
$$

The solution for the second equality of Eq. (A2) in the external potential of Eq. (3) is given by

$$
u(z)=\left\{\begin{array}{lc}
a_{1} \exp \left(k_{1} z\right)+a_{2} \exp \left(-k_{1} z\right) & -H_{1} \leq z \leq-a / 2 \\
b_{1} \sin \left(k_{0} z\right)+b_{2} \cos \left(k_{0} z\right) & -a / 2<z<a / 2 \\
c_{1} \exp \left(k_{2} z\right)+c_{2} \exp \left(-k_{2} z\right) & a / 2 \leq z \leq H_{2}
\end{array}\right.
$$

where $u(z)$ is the eigenfunction of Eq. (A2); $a_{1}, a_{2}, b_{1}, b_{2}, c_{1}$ and $c_{2}$ are constants to be determined by the boundary condition and the continuity of $u(z)$ and its first-order derivative. The boundary condition is given by

$$
u\left(-H_{1}\right)=u\left(H_{2}\right)=0
$$

The continuity of $u(z)$ and its first-order derivative require that

$$
\begin{aligned}
& a_{1} \exp \left(-k_{1} a / 2\right)+a_{2} \exp \left(k_{1} a / 2\right)=-b_{1} \sin \left(k_{0} a / 2\right)+b_{2} \cos \left(k_{0} a / 2\right) \\
& k_{1} a_{1} \exp \left(-k_{1} a / 2\right)-k_{2} a_{2} \exp \left(k_{1} a / 2\right)=k_{0} b_{1} \cos \left(k_{0} a / 2\right)+k_{0} b_{2} \sin \left(k_{0} a / 2\right) \\
& c_{1} \exp \left(k_{2} a / 2\right)+c_{2} \exp \left(-k_{2} a / 2\right)=b_{1} \sin \left(k_{0} a / 2\right)+b_{2} \cos \left(k_{0} a / 2\right) \\
& c_{1} k_{2} \exp \left(k_{2} a / 2\right)-k_{2} c_{2} \exp \left(-k_{2} a / 2\right)=k_{0} b_{1} \cos \left(k_{0} a / 2\right)-k_{0} b_{2} \sin \left(k_{0} a / 2\right) .
\end{aligned}
$$


Eq. (A6) yields

$$
\frac{k_{0} \tanh \left[k_{1}\left(H_{1}-a / 2\right)\right] \cos \left(k_{0} a\right)+k_{1} \sin \left(k_{0} a\right)}{-k_{0} \tanh \left[k_{1}\left(H_{1}-a / 2\right)\right] \sin \left(k_{0} a\right)+k_{1} \cos \left(k_{0} a\right)}=-\frac{\tanh \left[k_{2}\left(H_{2}-a / 2\right)\right]}{k_{2}} k_{0} .
$$

From Eq. (A7), we can obtain the eigenvalues of energy $E$ in the region $\left(-\varepsilon_{0}, 0\right)$. The eigenfunction is given by

$$
u(z)=\left\{\begin{array}{cc}
a^{\prime} \sinh \left[k_{1}\left(H_{1}+z\right)\right] / \cosh \left[k_{1}\left(H_{1}-a / 2\right)\right] & -H_{1} \leq z \leq-a / 2 \\
b_{1}^{\prime} \sin \left(k_{0} z\right)+b_{2}^{\prime} \cos \left(k_{0} z\right) & -a / 2<z<a / 2 \\
c^{\prime} \sinh \left[k_{2}\left(H_{2}-z\right)\right] / \sinh \left[k_{2}\left(H_{2}-a / 2\right)\right] & a / 2 \leq z \leq H_{2}
\end{array}\right.
$$

where

$$
\begin{aligned}
& b_{1}^{\prime}=-a^{\prime} \tanh \left[k_{1}\left(H_{1}-a / 2\right)\right] \sin \left(k_{0} a / 2\right)+a^{\prime} k_{1} \cos \left(k_{0} a / 2\right) / k_{0} \\
& b_{2}^{\prime}=a^{\prime} \tanh \left[k_{1}\left(H_{1}-a / 2\right)\right] \cos \left(k_{0} a / 2\right)+a^{\prime} k_{1} \sin \left(k_{0} a / 2\right) / k_{0} \\
& c^{\prime}=b_{1}^{\prime} \sin \left(k_{0} a / 2\right)+b_{2}^{\prime} \cos \left(k_{0} a / 2\right) .
\end{aligned}
$$

The unknown coefficient $a^{\prime}$ can be calculated from the normalization condition of the eigenfunction

$$
\int_{-H_{1}}^{H_{2}} u^{2}(z) \mathrm{d} z=1
$$

Similarly, for energy $E$ between zero and $\varepsilon_{1}-\varepsilon_{0}$, let

$$
k_{0}^{2}=6 \beta\left(E+\varepsilon_{0}\right) / l, \quad k_{1}^{2}=6 \beta\left(-E+\varepsilon_{1}-\varepsilon_{0}\right) / l, \quad k_{2}^{2}=6 \beta E / l .
$$

We can find another equation for eigenvalues similar to Eq. (A7)

$$
\frac{k_{0} \tanh \left[k_{1}\left(H_{1}-a / 2\right)\right] \cos \left(k_{0} a\right)+k_{1} \sin \left(k_{0} a\right)}{-k_{0} \tanh \left[k_{1}\left(H_{1}-a / 2\right)\right] \sin \left(k_{0} a\right)+k_{1} \cos \left(k_{0} a\right)}=-\frac{\tan \left[k_{2}\left(H_{2}-a / 2\right)\right]}{k_{2}} k_{0} .
$$

From Eq. (A11), we can obtain the eigenvalues of energy $E$ in the region $\left[0, \varepsilon_{1}-\varepsilon_{0}\right)$. The eigenfunctions are given by

$$
u(z)=\left\{\begin{array}{cc}
a^{\prime} \sinh \left[k_{1}\left(H_{1}+z\right)\right] / \cosh \left[k_{1}\left(H_{1}-a / 2\right)\right] & -H_{1} \leq z \leq-a / 2 \\
b_{1}^{\prime} \sin \left(k_{0} z\right)+b_{2}^{\prime} \cos \left(k_{0} z\right) & -a / 2<z<a / 2 \\
c^{\prime} \sin \left[k_{2}\left(H_{2}-z\right)\right] / \sin \left[k_{2}\left(H_{2}-a / 2\right)\right] & a / 2 \leq z \leq H_{2}
\end{array}\right.
$$

where the expressions for $b_{1}^{\prime}, b_{2}^{\prime}$ and $c^{\prime}$ are the same as those in Eq. (A9). For energy larger than $\varepsilon_{1}-\varepsilon_{0}$, let

$$
k_{0}^{2}=6 \beta\left(E+\varepsilon_{0}\right) / l, \quad k_{1}^{2}=6 \beta\left(E-\varepsilon_{1}+\varepsilon_{0}\right) / l, \quad k_{2}^{2}=6 \beta E / l .
$$


The equation for eigenvalues is given by

$$
\frac{k_{0} \tan \left[k_{1}\left(H_{1}-a / 2\right)\right] \cos \left(k_{0} a\right)+k_{1} \sin \left(k_{0} a\right)}{-k_{0} \tan \left[k_{1}\left(H_{1}-a / 2\right)\right] \sin \left(k_{0} a\right)+k_{1} \cos \left(k_{0} a\right)}=-\frac{\tan \left[k_{2}\left(H_{2}-a / 2\right)\right]}{k_{2}} k_{0} .
$$

From Eq. (A15), we can obtain the eigenvalues of energy $E$ larger than $\varepsilon_{1}-\varepsilon_{0}$. The eigenfunctions are given by

$$
u(z)=\left\{\begin{array}{lr}
a^{\prime} \sin \left[k_{1}\left(H_{1}+z\right)\right] & -H_{1} \leq z \leq-a / 2 \\
b_{1}^{\prime} \cos \left(k_{0} z\right)+b_{2}^{\prime} \sin \left(k_{0} z\right) & -a / 2<z<a / 2 \\
c^{\prime} \sin \left[k_{2}\left(H_{2}-z\right)\right] / \sin \left[k_{2}\left(H_{2}-a / 2\right)\right] & a / 2 \leq z \leq H_{2}
\end{array}\right.
$$

where

$$
\begin{aligned}
& b_{1}^{\prime}=a^{\prime}\left\{\frac{k_{1}}{k_{0}} \cos \left[k_{1}\left(H_{1}-\frac{a}{2}\right)\right] \cos \left(\frac{k_{0} a}{2}\right)-\sin \left[k_{1}\left(H_{1}-\frac{a}{2}\right)\right] \sin \left(\frac{k_{0} a}{2}\right)\right\} \\
& b_{1}^{\prime}=a^{\prime}\left\{\frac{k_{1}}{k_{0}} \cos \left[k_{1}\left(H_{1}-\frac{a}{2}\right)\right] \sin \left(\frac{k_{0} a}{2}\right)+\sin \left[k_{1}\left(H_{1}-\frac{a}{2}\right)\right] \cos \left(\frac{k_{0} a}{2}\right)\right\} \\
& c^{\prime}=b_{1}^{\prime} \cos \left(k_{0} a / 2\right)+b_{2}^{\prime} \sin \left(k_{0} a / 2\right) .
\end{aligned}
$$

\section{Energy and Entropy}

To calculate the energy $U$ and entropy $S$ of the system, we need to calculate the derivative of the partition function with respect to the reciprocal of Boltzmann factor $\beta$. To calculate the thermodynamic potential of the infinitely long polymer, we derive expression for the energy and entropy per unit polymer length. The energy and entropy are given by

$$
\begin{aligned}
& \beta U=-\frac{\beta}{Z L} \frac{\partial Z}{\partial \beta} \\
& S=k_{\mathrm{B}} L^{-1} \ln Z-\frac{k_{\mathrm{B}} \beta}{Z L} \frac{\partial Z}{\partial \beta} .
\end{aligned}
$$

From the partition function Eq. (1) and the bonding energy Eq. (2), we know that the zero point of the free energy i.e. $Z=1$ is obtained when the system is in the bulk, i.e., when external potential vanishes. This means that the free energy $F$ consists of two parts, one is from the bulk system and the other is from the contribution of the external potential.

$$
F(T)=F_{\text {bulk }}(T)+F_{\text {ext }}(T)=F_{\text {bulk }}(T)-k_{\mathrm{B}} T L^{-1} \ln Z .
$$


where $F_{\text {bulk }}$ and $F_{\text {ext }}$ are the free energy in bulk and in external field respectively. Eqs. (A18, A19) gives the variations of energy and entropy that result from the external potential including the oil-water interface and walls of the container.

For an infinitely long polymer, the free energy per polymer length is given by

$$
F_{\text {ext, } \infty}(T)=-k_{\mathrm{B}} T \lim _{L \rightarrow \infty} L^{-1} \ln Z=E_{0} .
$$

where $E_{0}$ is the eigenvalue of the ground state. Thus the energy and entropy of an infinitely long polymer are

$$
\begin{aligned}
& \beta U_{\infty}=\beta \frac{\partial \beta E_{0}}{\partial \beta} . \\
& S_{\infty}=-\frac{\partial F_{\text {ext }, \infty}}{\partial T}=k_{\mathrm{B}} \beta \frac{\partial \beta E_{0}}{\partial \beta}-k_{\mathrm{B}} \beta E_{0} .
\end{aligned}
$$

For a system without interface, the derivation is similar and simpler. When $H_{1}=H_{2}=H$, the eigenfunctions are given by

$$
u_{k}(z)=\left\{\begin{array}{ll}
\frac{1}{\sqrt{H}} \sin \frac{(n+1) \pi z}{H}, & k=2(n+1) ; \\
\frac{1}{\sqrt{H}} \cos \frac{\left(n+\frac{1}{2}\right) \pi z}{H}, & k=2 n+1 ;
\end{array} \quad n=0,1,2, \ldots .\right.
$$

The eigenvalues are given by

$$
\beta E_{k} / l=k^{2} \pi^{2} /\left(24 H^{2}\right) .
$$

The partition function is then given by

$$
Z=\sum_{n=0}^{+\infty} \frac{4 H}{\left(n+\frac{1}{2}\right)^{2} \pi^{2}} \exp \left(-\frac{L l(2 n+1)^{2} \pi^{2}}{24 H^{2}}\right) .
$$

The configurational partition function with no interface is independent of temperature. Thus walls of the container will not influence the energy according to Eqs. (A18, A19) but they influence the entropy. The entropy of an infinitely long polymer with no interface is given by

$$
S_{\infty}=-k_{\mathrm{B}} \pi^{2} l /\left(24 H^{2}\right) .
$$

If the width of the system is very large, the effect of the container walls becomes negligible. 